\title{
Examining the Relationship between a Structured Reading Framework and Students' Critical Thinking Ability within an Argument-Based Inquiry Approach
}

\author{
Jeong-yoon Jang* $\cdot$ Jeonghee Nam \\ University of Iowa, IA, USA $\cdot{ }^{1}$ Pusan National University
}

\begin{abstract}
This study examined how a Structured Reading Framework (SRF) is related to improving students' critical thinking ability in an argument-based inquiry approach, called the Science Writing Heuristic (SWH) approach. A total of $758^{\text {th }}$ graders participated in the study, with 34 in the control group and 41 in the treatment group. The gains in critical thinking skills were compared between two groups, and relationships among the components of the reading framework and the critical thinking skills were explored at the group level. Result indicates that the treatment group who used the SRF had larger gains in critical thinking scores than control group who used the Original Reading Framework (ORF). In addition, results show that the correlations between Reading Framework (RF) components and critical thinking scores are statistically significant in the treatment group, while no correlations exist in the control group. It appears that using the SRF have an impact on developing students' critical thinking ability by providing a scaffold to assist argumentation practice.
\end{abstract}

Key words: Reading Framework, Argumentation, Writing-to-learn, Critical thinking, Scaffold, Science Writing Heuristic (SWH) approach

\section{Introduction}

A major goal of the science literacy movement is that students be able to use science knowledge to think critically about important decisions that affect their personal and political well-being (Fensham, 2004; Organization for Economic Cooperation and Development, 2003). Science instruction is rooted in these goals of scientific literacy and is mirrored in Science Curriculum of Korea, which emphasize the importance for elementary and secondary students to make reasonable decisions as scientifically literate individuals. Success will result in students becoming literate members of the society who are able to think critically using scientific argumentation processes. Getting students to become critical thinkers engaged with highorder cognitive activity within science is not easily achieved; students need structured support to become proficient at using the appropriate reasoning strategies (Bransford et al., 1999).

A recent movement in science education has focused on the use of argumentation practices to engage students in thinking more deeply about science concepts (Osborne et al., 2004). Argumentation in science education requires students to construct and evaluate scientific arguments and to reason scientifically (Osborne, 2010). In a theoretical argument, Ford (2008) highlighted the importance of the dialectic between construction and critique in scientific reasoning and practice. He emphasized the importance of being a critique of science concepts: that is, "construction without appropriate critique would not result in the creation of new scientific knowledge" (Ford, 2008, p.410). Students need to be completely involved in an argumentation process that entails both constructing and critiquing knowledge in order to maximize their learning

*Corresponding author: Jeong-yoon Jang (jeongyoon-jang@uiowa.edu)

**Received on 16 October 2012, Accepted on 9 January 2013 
(Ford \& Forman, 2006). This point of view is supported by Mercier and Sperber's (2011) argument that there is "an asymmetry between the production of arguments and the evaluation of arguments"(p.72). Mercier (2001) pointed out that arguing-finding and evaluating argument - is the very function of reasoning. However, there is very little critique as evaluation occurs (Mercier \& Sperber, 2011). Ford (2008) argued that general teaching approaches have been without the critique function of scientific practice, thus students are not engaged in really grasping the practice of science. In traditional science classrooms, students rarely have opportunities to discuss, debate, construct and critique arguments for their ideas. As Ford and Forman (2006) argued, students must be given the opportunity to critique and evaluate scientific argument, and they often struggle with how to critique. According to Mercier and Sperber (2011), confirmation bias tends to occur in the production of arguments, and appears to restrict reasoning. When people reason on their own, they typically tend to look for arguments that have evidence supporting their own claim (Evans, 2007; Mercier \& Sperber, 2011). Osborne (2010) argued that students tend to adopt reasoning strategies with a confirmatory bias rather than using logical criteria. The confirmation bias is one of the main obstacles to be overcome in science education (Grcia-Mila and Andersen, 2008). It is important for students to understand that science argument is not just about their ideas but how their ideas compare to what is currently known and how they can align their views with current, accepted explanations (Hand, 2008). Thus this leads to questions: How do we enable students to move toward more critique, beyond the confirmation bias? One way to help students may be through scaffolding.

Scaffolding is viewed as providing enough support in order for learners to succeed in more complex tasks, and thereby to extend the range of experiences from which they can learn (Davis \& Linn, 2000; Reiser et al., 2001). By recognizing the importance of science argument as a critical component of knowledge construction, researchers have suggested that explicitly taught, through the provision of suitable activity, support, and modeling does improve students' scientific reasoning, and argument (Osborne, 2010; Simon et al., 2006). According to Cavenetto (2010), an immersive intervention, which emphasizes embedded science argument within the context of doing inquiry, facilitated argument through the use of scaffolding prompts and cognitive conflict. That is, by using the scaffolds as a guide for completing the science inquiry, students are required to both construct understanding and build their understanding around an argument framework (Cavenetto \& Hand, 2012). Guided activities, such as writing through questioning, notetaking, sorting, and peer review help students gain a greater depth of understanding of science inquiry. All of these activities involve use of language and writing-to-learn strategies.

Current efforts in science education stress the need for writing-to-learn strategies in science classrooms (Yore et al., 2003). Writing-to-learn strategies are viewed as being critical in the process of helping students understand science as a discipline and constructing rich understandings of the science concepts being studied. Importantly, these strategies are based on incorporating authentic writing tasks which extend students' needs to engage with the demands of science, rather than seeing writing as note-taking, fill-in-the-blank, or completethe-sentence type of exercises (Prain \& Hand, 1996). Writing to learn tasks incorporate the need for students to access canonical science knowledge and engage the nature of science, and their epistemologies and reasoning strategies as a framework to build understanding (Hand, Prain, Lawrence, \& Yore, 1999). Several studies show that incorporating writing-to-learn activities in science classrooms promotes science conceptual understanding (Bangert-Drowns et al., 2004; Gunel et al., 2009; Hand et al., 2004). 
Hand et al. (1999) argued that explicit writing tasks and instruction embedded in the authentic context of scientific inquiry should be provided as an integral part of science courses. In other word, students need to be provided with structured support and explicit instruction on how to transform their conceptual network into arguments and explanations. One example is the Science Writing Heuristic (SWH) approach.

The SWH approach developed by Hand and Keys (1999) is an argument-based inquiry approach to science instruction requiring students to negotiate their understanding of science concepts by engaging in the practices of science. Students both construct and critique science knowledge through processes that include the posing of questions, the construction of claims and the communication of evidence to support these claims. The SWH approach encourages students to think critically and reason logically as they negotiate understanding through talking, reading and writing. This negotiation involves a social process in which personal understanding is publically displayed and negotiated, a key component in developing scientific literacy. In particular, the SWH approach integrates the disciplines of writing and reading during science investigations to create an authentic scientific inquiry environment in which dialogical interactions replace traditional didactic approaches (Hand, 2008). When the full sequences of the SWH approach are implemented, students are engaged in a more complete process of scientific inquiry. By using a SWH approach, students are required to not only construct understanding, but also build their understanding around an argument (Hand, 2008). Students are not told explicitly how to do the experiments, but rather, they are required to be more active in generating and answering questions. In a SWH approach, students are required to be involved in completing a distinct writing activity with the SWH templates during scientific inquiry activities.
Taken as a whole, this present study focused on a written scaffold to promote the argumentative practice of construction and critique through a writing-to-learn activity in an argument based inquiry approach. In particular, this study investigated how using a written scaffold is related to improving critical thinking ability. Given the purpose of this study, the research proposed here took place within the context of the SWH approach. The following research questions guided the design of the study:

1. Does the use of the Structured Reading Framework embedded in the SWH template promote greater gains in students' critical thinking skills than the Original Reading Framework?

2. Given the difference of the gain growth between two groups, how do the two groups differ in the relationships between the components of the Reading Framework and critical thinking ability?

\section{Method}

\section{Participants}

Participants were $8^{\text {th }}$ grade students taught by a teacher in pre-existing classes at a middle school located in the urban area around the biggest city in South Korea. A total of 75 students participated in the study, with 34 in the control group (one class) and 41 in the treatment group (one class).

\section{Research Context}

This study was situated within s SWH classroom context. The SWH approach provides students with a heuristic template to guide science activity and reasoning through writing. The student template of the SWH is designed to help them construct scientific knowledge within a scientific inquiry (Keys et al., 1999). The SWH template consists of the following seven phases: 
Beginning ideas (what are my questions?), tests (what did I do?), observation (what can I see?), claims (what can I claim?), evidence (how do I know? Why am I making these claims?), reading (how do my ideas compare with others?), and reflection (how have my ideas changed?). The SWH student template is useful in scaffolding students' conceptual understanding, writing, and critical thinking (Hohenshell \& Hand, 2006; Rudd et al., 2001). In this study, the focus was on the reading phase of the $\mathrm{SWH}$ approach. In addressing the question (how do my ideas compare with other?) of the reading phase, students are required to seek information from other various sources, such as textbooks, science magazines, journals, scientists, or through the internet, and are provided opportunities to make the connection between their ideas and the science ideas. However, in a study by Jang (2011), she indicated that middle school students who used the original reading framework within the SWH template struggled to see other point of view and to see coherence of their argument, and appeared to need more structured scaffold. For this study, a Structured Reading Framework (SRF) for this particular component of the reading framework was used for the treatment group.

While the ORF has just simple statement that may guide note-taking during the reading activity and no guiding question, the SRF has prompts such as source, information, and comparison. As Osborne (2010) pointed out, the individual develops new understanding through a cognitive process of comparison and contrast. There is a need for students to engage in going beyond the confirmation bias, and seeing coherent checking on their arguments (Jang \& Hand, 2012). Therefore, the SRF attempted to promote two essential features: one is going back to claim and evidence to compare developed ideas, and the second is going forward to examine if these claims and evidence, and comparisons are coherent. Jang and Hand (2012) suggested that students' active coherent checking using a more structured written scaffold allows them to develop their argumentation skills. The SRF is based on two critical stages. The first stage is centered on recording information from a source. That is, students go to test against scientifically accepted ideas which move beyond their ideas, and then move beyond confirmation bias (Jang \& Hand, 2012). The second stage is focused on requiring students to compare their recorded information to their claims and evidence, and the beginning ideas they have previously generated. This promotes not just comparison, but also coherence of arguments (Jang \& Hand, 2012). These two tasks are intended to assist the reading phase by supporting students in negotiating with what they read from various sources, and to sharpen their conceptual understanding of the big ideas of the unit. This requires them to compare ideas from other sources to their own constructed ideas. In other words, the students are required to both synthesize and analyze a number of potentially competing ideas. A sample of the SRF was provided in Appendix A.

\section{Instrument}

The Cornell Critical Thinking Test (CCTT) (Level X; Ennis et al., 2005) examined the change of students' critical thinking abilities at the beginning and the end of the intervention. The CCTT measures critical thinking ability of students in grades $4-14$. The test consists of four sections with a total of 76 multiple-choice items. The questions assess critical thinking skills in general, without specific academic content, and there is no requirement of science content to complete this test. The test contains four subscales of critical thinking: Induction, Deduction, Credibility \& Observation, and Assumption. These subscales are closely aligned with the thinking skills necessary to engage in the SWH approach. As such, the researchers believed that the Cornell Critical Thinking test 
adequately reflected the thinking skills required of the students.

\section{Research Design and Procedure}

A quasi-experimental design was used, and $8^{\text {th }}$ grade students in the urban area around the biggest city in South Korea participated. The Cornell Critical Thinking Test was administrated to all participants at the beginning and the end of a school year as a pre-test and post-test, respectively. All students from both treatment and control group used the SWH student templates to guide their written work and completed the SWH student templates during the SWH investigations. The only difference between the comparison groups was in the reading phase within the SWH template. The control group had an original reading framework for the reading phase, while the treatment group had a structured reading framework.

\section{Data Collection}

There were two main data sources used for this study. First, all students' completed SWH reports were collected. The actual number of student writing samples collected varied over the 4 units. The number of writing samples collected from each unit by two groups varied because of factors related to attendance and failure to complete the activities. For this reason, in order to maintain uniformity and consistency, the SWH report from last unit among 4 SWH reports produced by students during the SWH investigations were selected. In total, 75 writing samples was collected and analyzed in this study. Second, students' pre-, and post-CCTT score were collected.

\section{Data Analysis}

\section{1) Analyzing Student Writings}

Analytical framework developed by Jang (2011) was used to evaluate the student writing samples of the reading framework (see Table 1). The analytical framework for the reading framework includes two components: the use of information, and the quality of comparison.

Two raters who were graduate students in science education independently scored students' writing samples. The reliability for all components of the reading framework tested by inter-rater reliability using Pearson correlation coefficient, and internal consistency using Cronbach's $\alpha$. Internal consistency were .787, and Pearson's correlation coefficients were acceptable ( $r=.836$ to $1.000, p<.001)$.

\section{2) Effect Size Calculation}

In terms of effect sizes, the growth score from pre- to post-test of each group was estimated using Cohen's d. Cohen (1992) provides a standard categorical scheme for classifying effect size calculations, with effect sizes below .20 categorized as negligible, effect sizes between .20 and .49 categorized as small, effect sizes of .50 to .79 as medium, and effect sizes of

\section{Table 1}

Analytical Frameworks for the Reading Framework

\begin{tabular}{|c|c|c|}
\hline \multicolumn{3}{|c|}{ The Reading Framework } \\
\hline Component & Criteria & Score Scale \\
\hline \multirow[t]{2}{*}{ Use of Information } & \multirow{2}{*}{$\begin{array}{l}\text { - Is the information from the source credible? } \\
\text { - Is the information from the sources related to the } \\
\text { question/big idea proposed by students? }\end{array}$} & $0-1$ \\
\hline & & $0-3$ \\
\hline \multirow[t]{2}{*}{ Quality of Comparison } & \multirow{2}{*}{$\begin{array}{l}\text { - Do students recognize what/how information is } \\
\text { similar/different to their claim \& evidence/beginning idea? } \\
\text { - Is the comparison accurate and adequate? }\end{array}$} & $0-3$ \\
\hline & & $0-3$ \\
\hline
\end{tabular}


.80 and greater as large. These categories were utilized in this study.

\section{3) Partial Correlation}

Using partial correlation analysis controlling for the pre-CCT score, the relationships among the components of the reading framework and five subscales of critical thinking were investigated at the group level.

\section{Result}

Results are reported in the order the research questions were posed. First, whether the treatment group which used the structured reading framework embedded in the $\mathrm{SWH}$ template had greater gain in the CCTT compared to the control group was determined. Next, using partial correlation analysis controlling for the pre-CCTT total score, relationships among the components of the reading framework and the CCTT were explored at the group level.

\section{Group Differences on the CCTT score}

In addressing the first research question, effect sized differences were calculated between the two groups. As shown in Table 2, although both groups had a significant gain from pre-to post-test, the treatment group had a greater effect size gain than the control group. The control group had a significant gain in total score with small effect size (Cohen's $d$ size $=$ 0.455), while the treatment group also had a significant gains in total score, but with a medium effect size (Cohen's $d$ size $=0.704$ ). In particular, for the subscales of deduction and assumption, while the control group had a significant gain from pre- to post-test with a small effect size (Cohen's $d$ size $=0.428$ for the deduction, Cohen's $d$ size $=0.221$ for assumption), the treatment group had a significant gain with a medium effect size (Cohen's $d$ size $=0.613$ for the deduction, Cohen's $d$ size $=0.556$ for assumption)

\section{Relationships among the Components of the Reading Framework and the CCTT}

The second research question examined the relationships between the components of the Reading Framework (RF) and the critical thinking ability. The degree of relationships

\section{Table 2}

Descriptive Statistic of CCTT, and Cohen's d Effect Size on Gain Score from Pre-test to Post-test by Group

\begin{tabular}{|c|c|c|c|c|c|c|c|c|}
\hline & \multicolumn{4}{|c|}{ Control (N=34) } & \multicolumn{4}{|c|}{ Treatment $(\mathrm{N}=41)$} \\
\hline & Mean & $\mathrm{SD}$ & Cohen's $d$ & Scale & Mean & $\mathrm{SD}$ & Cohen's $d$ & Scale \\
\hline Pre-Induction & 12.15 & 4.580 & 0.361 & Small & 13.32 & 3.684 & 0.355 & Small \\
\hline Post-Induction & 13.74 & 4.231 & & & 14.66 & 3.864 & & \\
\hline Pre-Deduction & 11.50 & 5.264 & 0.428 & Small & 12.63 & 3.878 & 0.613 & Medium \\
\hline Post-Deduction & 13.62 & 4.625 & & & 15.02 & 3.915 & & \\
\hline $\begin{array}{l}\text { Pre-Observation } \\
\text { \&Credibility }\end{array}$ & 10.94 & 4.299 & 0.461 & Small & 11.24 & 2.826 & 0.485 & Small \\
\hline $\begin{array}{l}\text { Post-Observation } \\
\text { \&Credibility }\end{array}$ & 12.68 & 3.169 & & & 12.61 & 2.827 & & \\
\hline Pre-Assumptions & 3.76 & 2.104 & 0.221 & Small & 3.90 & 1.814 & 0.556 & Medium \\
\hline Post-Assumptions & 4.24 & 2.230 & & & 5.00 & 2.133 & & \\
\hline Pre-Total & 38.74 & 12.410 & 0.455 & Small & 41.05 & 8.047 & 0.704 & Medium \\
\hline Post-Total & 43.74 & 9.327 & & & 46.54 & 7.544 & & \\
\hline
\end{tabular}


between the components of the RF and CCTT is shown in Table 3 at the group level. For the treatment group, the correlation between the $\mathrm{RF}$ total score and CCTT total score is .464 $p<.01$ ), which is statistically significant at the 0.01 level. However, no correlation existed in the control group. In particular, while the Quality of Comparison component score of the RF was significantly and positively correlated with the CCTT total score in the treatment group ( $r=$ .440, $p<.01)$ this was not seen in the control group. Further, in the treatment group, the Use of Information component score was significantly correlated with the deduction score of the CCTT ( $r=.491, p<.01)$, and the Quality of Comparison component of the Reading Framework was significantly correlated with the induction score of the CCTT $(r=.400, p<.05)$. In contrast, in terms of correlation between the components of the RF and the subsections of the CCTT, there was no significant correlation in control group.

\section{Discussion}

We would like to reiterate that this study is focused on the students' writing after they have completed their reading that occurs within the Reading Framework. The intention of the Reading Framework in this study was to prompt students to use information from their reading in determining how their claims and evidences were aligned to scientific knowledge. The researchers emphasize that the Reading Framework, which is looking at the written product, is the writing, not the reading.

The effect size differences indicated that both groups had a significant gain from pre-to posttest, the treatment group had a greater effect size gain than the control group. Recent research related to the SWH approach has indicated that students using the SWH approach experience a significant improvement in critical thinking skills compared to students who participate in more traditional approaches to science instruction ( Hand et al, 2012; Shelley et al, 2012; Taylor et al., 2012). In this regard, when considering this study was conducted within SWH approach, effect size gains for both groups were expected. However, more importantly, the treatment group of students who used the Structured Reading Framework (SRF) had greater growth rates in critical thinking scores as measured by effect sizes than those of the control group. That is, the value of using the SRF can be seen as important precursor to developing students' critical thinking ability.

The second research question aimed to examine how different two groups are, and to

\section{Table 3}

Partial Correlations between the Components of the Reading Framework and the Critical Thinking Test by Group

\begin{tabular}{|c|c|c|c|c|c|c|}
\hline & \multicolumn{3}{|c|}{ Control Group (N=34) } & \multicolumn{3}{|c|}{ Treatment Group (N=41) } \\
\hline & UI & $\mathrm{QC}$ & RF Total & UI & $\mathrm{QC}$ & RF Total \\
\hline Induction & .077 & .109 & .112 & -.016 & $.400 *$ & $.334^{*}$ \\
\hline Deduction & -.169 & -.054 & -.114 & $.491 * *$ & .290 & $.410 * *$ \\
\hline $\begin{array}{l}\text { Observation \& } \\
\text { Credibility }\end{array}$ & .066 & .033 & .053 & .091 & .195 & .196 \\
\hline Assumptions & -.177 & -.117 & -.162 & .277 & .212 & .273 \\
\hline CCTT Total & -.017 & .034 & .016 & .272 & $.440 * *$ & $.464^{* *}$ \\
\hline
\end{tabular}

**. Correlation is significant at the .01 level (2-tailed)

*. Correlation is significant at the .05 level (2-tailed) 
understand the relationships between the components of the Reading Framework $(\mathrm{RF})$ and the critical thinking ability, given the difference between two groups. While it is understood that a significantly positive correlation is not indicative of a causal relationship, it is evidence that a positive relationship exists between the variables. These results provide possible evidence to support the effect size calculation result discussed earlier, and to explain why there is a difference between the two groups on the magnitude of the effect of the CCTT.

Result of this study showed that in the treatment group, RF total score is significantly correlated with CCTT total score. In contrast, there was no significant correlation in the control group. This result indicates that what information students use, and how they use their information with the RF are positively related to developing their critical thinking ability. Stated differently, the correlation between these two components suggests that the better information students have, and the better they are able to compare their previous knowledge with new scientific knowledge they have just read, and the better they develop their critical thinking ability. Given that the function of the RF of the SWH template promotes argumentative explanation (Jang, 2011), it can be argued that a good job in argumentative explanation writing helps students develop critical thinking ability. In addition, it appears that the SRF promotes this relationship because this relationship did not exist with students in the control group. That is, the lack of prompts in the ORF that the control group used may not be helpful in building a strong positive relationship. When considering that the treatment group students who used the SRF had larger gain in the CCTT total score than those who used the $\mathrm{ORF}$, it is speculated that the SRF provided significant support structure in developing critical thinking ability.

In particular, while the Quality of Comparison component score of the RF was significantly and positively correlated with the CCTT total score in the treatment group, this was not seen in the control group. Since the Quality of Comparison score reveals how well students compare and contrast information from the reading source with their previous understanding and their own claims and evidence, this result appears to suggest if students compare well, and have better coherence on argument structure, their critical thinking ability will be stronger. The second prompt of the SRF of the treatment group provides students with the opportunity to evaluate and critique their own ideas by comparing and contrasting previously generated claim and evidence with new science ideas gained from information gathering. This leads to not only building scientific knowledge (concept knowledge), but also improving structure of argument by coherence checking of their argument (Jang \& Hand, 2012). Given that the comparison prompt of the SRF promotes an evaluative and critiques process, and is critical to develop structure of argument, its significant correlation with the CCTT total score in the treatment group seems expected. Further, while this relationship was positively significant in the treatment group, no relationship was found in the control group. In this regard, considering that the treatment group performed better than the control group for the CCTT total score, the researcher would suggest that the comparison prompt of the SRF is crucial to develop students' critical thinking capability.

In terms of correlation between the components of the RF and the subsections of the CCTT, in the treatment group, the Use of Information component score was significantly correlated with the deduction score of the CCTT, and the Quality of Comparison component of the RF was significantly correlated with the induction score of the CCTT. The items of the deduction and induction of the CCTT emphasizes the importance of reasoning (Ennis, 2005). The Use of Information component score indicates how well students use credible information and 
how well the information from the reading sources is connected to the big idea and their claims and evidence. This relationship implies that if students use more credible information, which is more connected the big idea, they have better deduction score on the CCTT. In other words, the ability to determine more reliable and appropriate information is closely associated with inductive reasoning. Further, this relationship existed only in the treatment group, inferring that the prompts in the SRF promoted this relationship. On the other hand, the correlation between the Quality of Comparison component of the RF and the induction score of the CCTT in the treatment group implies that a student who does more active coherence checking on scientific knowledge and structure of argument is likely to relate to inductive reasoning. In addition, this relationship appears to be promoted by the prompt SRF.

\section{Implications}

The main implication for teachers is the realization of the importance of the writing activity scaffolding the reading as an argumentation practice to negotiate with other scientific information, and promote critical thinking ability. Students need practice to go beyond confirmation bias and evaluate their ideas in order to communicate in an accepted scientific manner as a fundamental part of doing inquiry. Thus, science teachers should provide students with a more structured scaffold which guides students in the process of communication with scientific information and constructing knowledge in logical ways. The results of this study indicate that the SRF enables students to have more information, make better comparisons, and then use information better. Therefore, we need to help teachers understand both what the scaffold is and how to implement the scaffold.

The SWH template consists of seven phases. As the last phase of the SWH template, the reflection section builds on and follows the reading activity to provide writing experience that requires students to examine if and how their idea have changed though the SWH. In addition, in a SWH approach, students are required to be involved in completing two distinct writing activities that have different purposes - the SWH templates during scientific inquiry activities and summary writing tasks at the end of unit. When considering the series of students' writing-to-learn activities, there is a need to examine the direct and indirect effects of the Reading Framework on students' performance with sophisticated modeling and methods of analysis techniques.

Due to its small sample size, this study was not able to conduct a regression analysis to estimate causal effects. This should be examined in the near future to gain a better understanding of how the use of the structured scaffold is related to critical thinking ability. However, we believe that this study provides additional information to a developing body of research with the writing-to-learn activity based on argument based inquiry approach.

\section{References}

Bangert-Drowns, R.L., Hurley, M.M., \& Wilkinson, B. (2004). The effects of school-based writing-to- learn interventions on academic achievement: A meta-analysis. Review of Educational Research, 74, 29-58.

Bransford, J., Brown, A. L., Cocking, R. R., \& National Research Council. (2000). How people learn: Brain, mind, experience, and school. Washington, D.C.: National Academy Press.

Cavagnetto, A. R. (2010). Argument to foster scientific literacy; a review of argument interventions in $\mathrm{K}-12$ science contexts. Review of Educational Research, 80(3), 336-371.

Cavagnetto, A. R., \& Hand, B. (2012). The Importance of embedding argument within science classrooms : Perspectives on scientific argumentation, Springer, Part 1, 39-53. 
Davis, E. A.,\&Linn, M. C. (2000). Scaffolding students' knowledge integration: Prompts for reflection in KIE. International Journal of Science Education, 22, 819-837.

Ennis, R. H., Millman, J., \& Tomka,T. N. (2005). Manual: Cornell Critical Thinking Tests. Pacific Grove,CA: Midwest.

Evans, J. St. B. T. (2007) Hypothetical thinking: Dual processes in reasoning and judgment. Psychology Press.

Fensham, P. J. (2004). Defining an identity: The evolution of science education as a field of research. Dordrecht, the Netherlands: Kluwer Academic Publishers.

Ford, M. J. (2008). Disciplinary authority and accountability in scientific practice and learning. Science Education, 92, 404-423.

Ford, M. J., \& Forman, E. A. (2006). Redefining disciplinary learning in classroom contexts. Review of Research in Education, 30(1), 1-32.

Garcia-Mila, M., \& Andersen, C. (2008). Cognitive foundations of learning argumentation. In S. Erduran \& M.P. Jimenez-Aleixandre (Eds.), Argumentation in science education. Recent developments and future directions (pp. 29-47). Dordrecht, Netherlands: Springer.

Gunel, M., Hand, B., \& McDermott, M. (2009). Writing for different audiences: Effects on high school students conceptual understanding of biology. Learning and Instruction, 19 (4), 354367.

Hand, B. (Ed.). (2008). Science inquiry, argument and language: A case for the Science Writing Heuristic. Rotterdam, The Netherlands: Sense.

Hand, B., \& Keys, C. (1999). Inquiry investigation. The Science Teacher, 66(4), $27 ? 29$.

Hand, B., Hohenshell, L., \& Prain, V. (2004). Exploring students' responses to conceptual questions when engaged with planned writing experiences: A study with Year 10 science students. Journal of Research in Science Teaching, 41(2), 186-210.

Hand, B., Prain, V., Lawrence, C, \& Yore, L.
(1999). A writing in science framework designed to enhance science literacy. International Journal of Science Education, 21(10), 1021-1035.

Hand, B., Shelley, M., Gonwa-Reeves, C., \& Baenzinger, J. (2012, March). Student learning through the Science Writing Heuristic: Iowa Test of Basic Skills, Cornell Critical Thinking Tests and classroom implementation. Paper presented at the Annual Meeting of National Association of Science Teachers, Indianapolis, Indiana.

Hohenshell, L. M., \& Hand, B. (2006). Writing-to-learn strategies in secondary school cell biology: A mixed method study. International Journal of Science Education, 28(2-3), 261-289.

Jang, J. (2011). The effect of using a structured reading framework on middle school students' conceptual understanding within the Science Writing Heuristic approach. Unpublished doctoral dissertation, The University of Iowa, Iowa city, IA.

Jang, J., \& Hand, B. (2012, March). The impact of using a scaffolded written framework on students' conceptual understanding. Paper presented at International Conference of the National Association for Research in Science Teaching, Indianapolis, IN.

Keys, C. W., Hand, B., Prain, V., \& Collins, S. (1999). Using the Science Writing Huerisitic as a tool for learning from laboratory investigations in secondary science. Journal of Research in Science Teaching, 36(10), 1065-1084.

Mercier, H. (2011). Looking for arguments. Argumentation, 26, 305-324.

Mercier, H., \& Sperber, D. (2011). Why do humans reason? Arguments for an argumentative theory. Behavioral and Brain Sciences, 34, 57-74.

Organisation for Economic Co-operation and Development. (2003). The PISA 2003 assessment framework: Mathematics, reading, science and problem solving knowledge and skills. Paris: Author.

Osborne, J. (2010). Arguing to learn in science: The role of collaborative, critical 
discourse. Science, 328, 463-466.

Osborne, J., Erduran, S., \& Simon, S. (2004). Enhancing the quality of argumentation in school science. Journal of Research in Science Teaching, 41, 994-1020.

Prain, V., \& Hand, B. (1996). Writing for learning in secondary science: Rethinking practices. Teaching and Teacher Education, 12(6), 609-626.

Reiser, B. J., Tabak, I., Sandoval,W. A., Smith, B. K., Steinmuller, F., \& Leone, A. J. (2001). BGuILE: Strategic and conceptual scaffolds for scientific inquiry in biology classrooms. In S. M. Carver \& D. Klahr (Eds.), Cognition and instruction: Twenty-five years of progress (pp. 263-305). Mahwah, NJ: Lawrence Erlbaum Associates, Inc.

Rudd, J. A., Greenbowe, T. J., Hand, B. M., and Legg. M. J. (2001). Using the Science Writing Heuristic (SWH ) to move toward an inquirybased laboratory curriculum: An example from physical equilibrium. Journal of Chemical Education, 78(12), 1680-1686.

Shelley, M., Gonwa-Reeves, C., Baenziger, J., Hand, B., \& Therrien, W. J. (2012, April). Student learning and inquiry-based science instruction: Testing effectiveness in a randomized trial. Paper presented at the Annual Meeting of the American Educational Research Association, Vancouver, BC.

Simon, S., Erduran, S., \& Osborne, J. (2006). Learning to teach argumentation: Research and development in the science classroom. International Journal of Science Education, 28, 235-260.

Taylor, J. C., Therrien, W. J., Kaldenberg, E., Watt, S., Chanlen, N., \& Hand, B. (2012). Using an inquiry-based teaching approach to improve science outcomes for students with disabilities: Snapshop and longitudinal data. Journal of Science Education for Students with Disabilities, 15(1), 10-22.

Yore, L. D., Bisanz, G. L., \& Hand, B. M. (2003). Examining the literacy component of science literacy: 25 years of language arts and science research. International Journal of Science Education, 25(6), 689-725.

Yore, L., \& Treagust, D. (2006). Current realities and future possibilities: Language and science literacy - empowering research and informing instruction. International Journal of Science Education, 28(2), 291-314. 


\section{Appendix A: A sample of Structured Reading Framework}

\section{힉생 읿기틀}

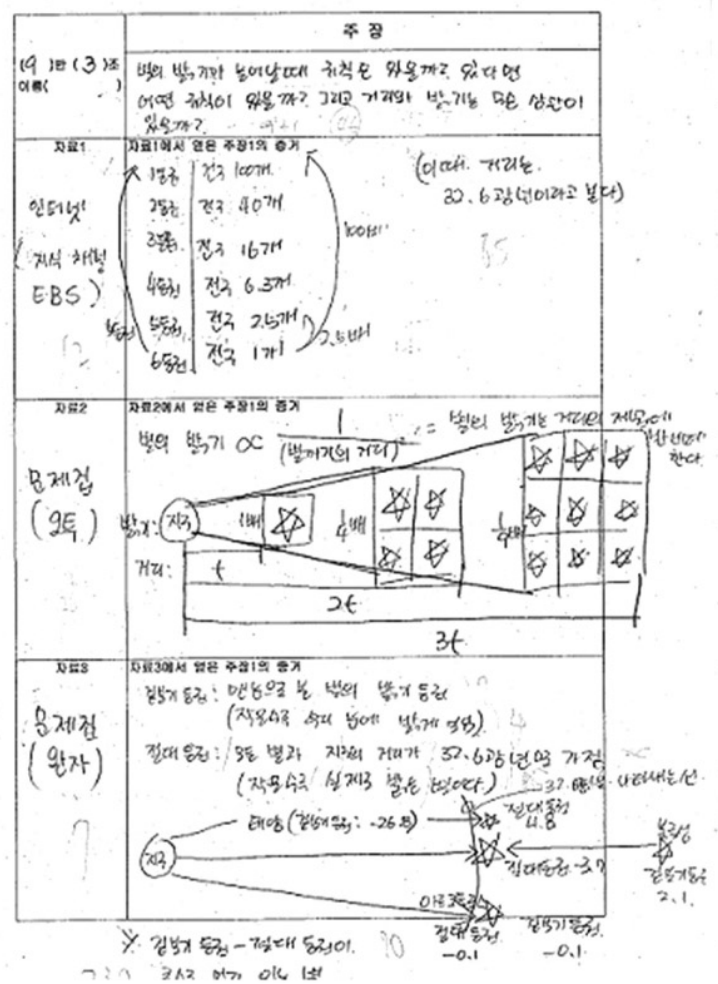

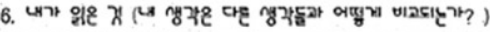

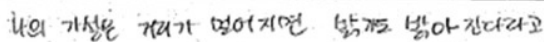

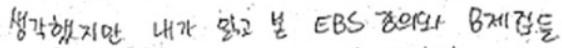

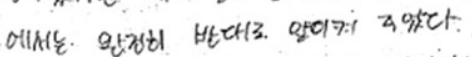

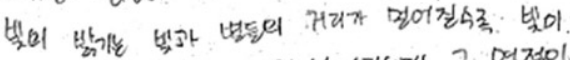

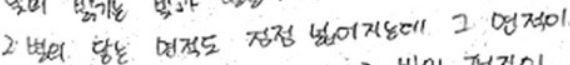

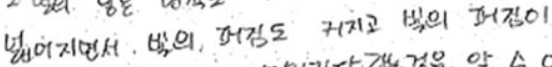

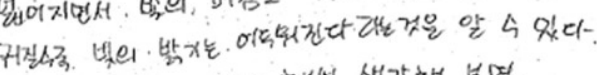

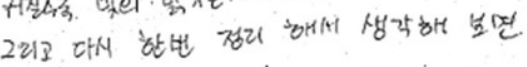

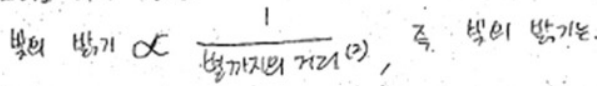

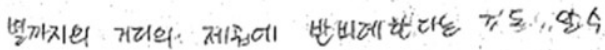

of 9 자.

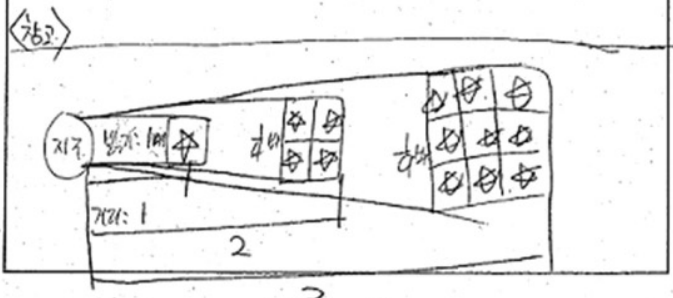

